\title{
SUBAFLORAMENTO DAS DISCORDÂNCIAS TURONIANA E CAMPANIANA NO SUL DA BACIA DE CAMPOS.
}

\author{
ALEXANDRE JOSÉ RICHTER*
}

\begin{abstract}
An accurate investigation of the calcareous nannoplankton content, recorded in the Albian-Maastrichtian marine section of 109 exploratory wells drilled by Petrobrás in the south part of Campos Basin, southeastern Brazil, allowed the construction of a refined biostratigraphic framework and the reconnaissance of two widespread discontinuities. Such stratigraphic breaks, here named Turonian Unconformity and Campanian Unconformity, correspond to gaps of sedimentation spanning these age intervals in this part of the basin. Subcrop maps of the unconformities show the geographic distribution of the traced biostratigraphic units. The zonal scheme used in this study was based on the vertical distribution of selected calcareous nannofossils recorded in the Brazilian marginal basins and recognized as biochronostratigraphically diagnostic forms of worldwide occurrence.
\end{abstract}

INTRODUÇÃo A Bacia de Campos, atualmente a mais importante província petrolífera do Brasil, desenvolve-se costa afora entre os paralelos do $21^{\circ}$ e $24^{\circ}$ de latitude sul. Possui área aproximada de $35.000 \mathrm{~km}^{2}$, até a cota batimétrica de $500 \mathrm{~m}$, e está limitada por duas feições estruturais: o Arco de Cabo Frio, no litoral norte fluminense, e o Arco de Vitória, no litoral sul do Espírito Santo. A área investigada situa-se no sul da bacia (Fig. 1).

A coluna estratigráfica acumulada em Campos alcança mais de 4.000 metros de possança e, de acordo com o ambiente em que foi depositada, pode ser dividida em duas seqüências distintas: uma seção basal, não-marinha, de idade pré-albiana, assentada diretamente sobre rochas cristalinas basálticas da crosta oceânica, e outra, sobrejacente à primeira, depositada em condições marinhas, a partir do Albiano até o Recente (Fig. 2).

Este trabalho objetiva divulgar os resultados de uma detalhada investigação bioestratigráfica realizada na seção cretácea marinha do sul da Bacia de Campos, utilizando nanofósseis calcários. Além do arcabouço bioestratigráfico obtido, são apresentados mapas de subafloramento de duas discordâncias que interrompem a seqüência estratigráfica Albiano-Maastrictiano.

MÉTOdos A pesquisa foi executada por meio do estudo, sob microscópio óptico, da associação nanoflorística contida em testemunhas e em amostras de calha. Estas, representativas de intervalos regulares de $15 \mathrm{~m}$, provenientes de 109 poços.

$\mathrm{O}$ material foi minuciosamente analisado, visando determinar, com a maior precisão possível, o topo e a base das diferentes unidades bioestratigráficas que integram a seqüência cretácea marinha perfurada em cada poço. Essas unidades foram definidas com base no nível de extinção ou na amplitude local de formas-guias já utilizadas nos zoneamentos de nanoplâncton calcário propostos por Troelsen \& Quadros (1971) e Thierstein (1976). Para melhor visualizar a amplitude dos hiatos que interrompem a seção estudada, inserimos, no zoneamento obtido, as biozonas que faltam, mas que se acham presentes em outras bacias da margem continental brasileira: zona Corollithion achylosum (informal) e zona Eiffellithus eximius (Fig. 3).

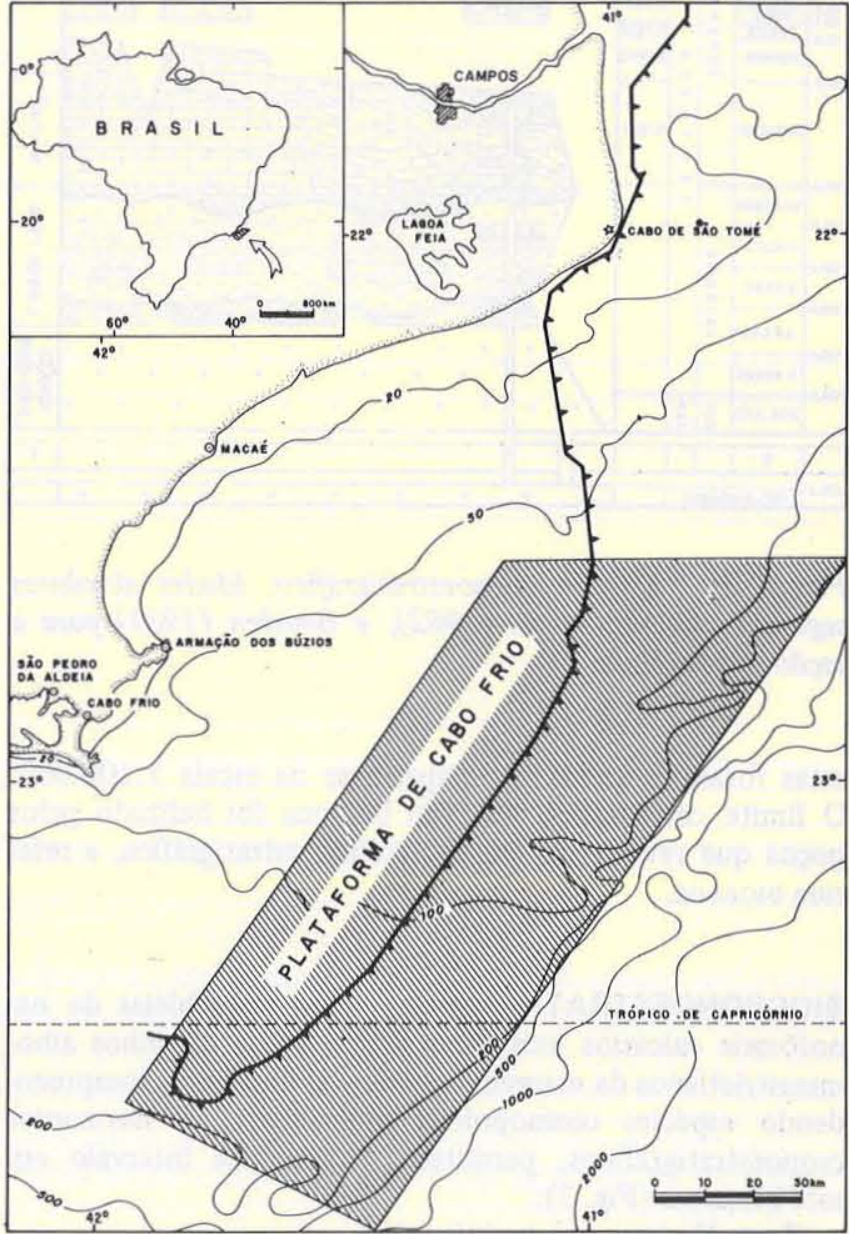

Figura 1 - Mapa de localização e área estudada

Para elaborar os mapas de subafloramento das discordâncias detectadas na seqüência estratigráfica estudada, foi necessário minucioso controle temporal e espacial das biozonas. Uma vez definidas em seus limites, identificadas por nomes, códigos alfanuméricos e cores convencionadas, 


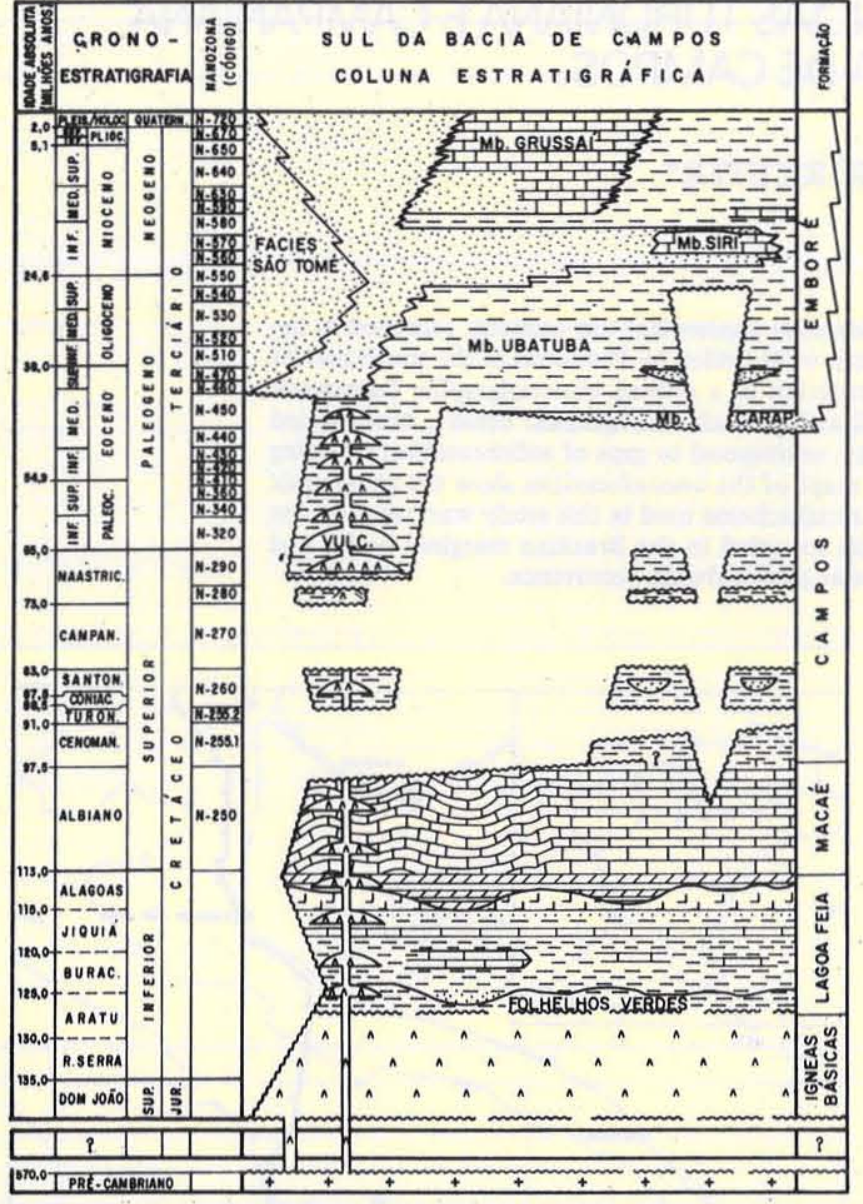

Figura 2 - Quadro cronoestratigráfico. Idades absolutas segundo Harland et al. (1982), e Beurlen (1981) para a seção não-marinha

estas foram lançadas em mapas-base na escala 1:100.000. O limite cartográfico de cada biozona foi balizado pelos poços que revelaram, em sua coluna estratigráfica, a referida biozona.

BIOCRONOESTRATIGRAFIA As assembléias de nanofósseis calcários existentes nos estratos marinhos albo-maastrictianos da margem continental brasileira, compreendendo espécies cosmopolitas indicadoras de horizontes cronoestratigráficos, permitem dividir esse intervalo em sete biozonas (Fig. 3):

- Zona Nannoconus truitti truitti

- Zona Podorhabdus albianus (informal)

- Zona Corollition achylosum (informal)

- Zona Marthasterites furcatus

- Zona Eiffellithus eximius

- Zona Tetralithus trifidus

- Zona Arkhangelskiella cymbiformis

No sul da Bacia de Campos, a ausência das biozonas Corollithion achylosum e Eiffellithus eximius denuncia a ocorrência de dois hiatos estratigráficos que interrompem a sequêencia Albiano Maastrictiano. Tais quebras caracterizam duas discordâncias regionais: a mais antiga, denominada Discordância Turoniana, e a mais nova, Discordância Campaniana.
Zoneamento Cretáceo Local ZONA Nannoconus trutti truitti $(N-250)$ A zona é definida pela amplitude local de Nannoconus truitti truitti.

Na Bacia de Campos, seu topo é assinalado no nível de extinção de Nannoconus spp., e a base coincide com o topo dos sedimentos não-marinhos e transicionais da Formação Lagoa Feia.

As espécies mais comuns no intervalo são: Nannoconus truitti truitti, Nannoconus spp., Watznaueria barnesae, Lithastrinus floralis e Eiffellithus turriseiffelli.

A biozona ocorre em toda a área mapeada e está parcialmente coberta por estratos da biozona informal Podorhabdus albianus (Fig. 4).

Os sedimentos desta biozona são marinhos, de idade Albiano.

ZONA INFORMAL Podorhabdus albianus (N-255.1) A zona é definida, no topo, pelo nível de extinção de Podorhabdus albianus e, na base, pela última ocorrência de Nannoconus spp.

A biozona é portadora de poucas espécies de nanofósseis e a forma-guia raramente está presente. A presença da zona, entretanto, é inferida sempre que, no sul da Bacia de Campos, é perfurada uma seção estratigráfica pobre em nanofósseis, limitada, no topo, por uma associação típica do Coniaciano-Santoniano ou mais nova e, na base, por uma assembléia característica do Albiano. Como o critério não oferece confiabilidade total, por se basear em evidência negativa, a presença da zona pode ser aferida lançando-se mão do zoneamento palinológico adotado pela Petrobrás. Se o intervalo em questão for portador do pólen Classopolis major, que define o "intervalo $\gamma$ " do zoneamento palinológico, fica caracterizada a presença da seção correspondente à zona $P$. albianus, pois ambos os fósseis possuem nível de extinção aproximadamente síncronos.

As espécies mais comuns que ocorrem nesta biozona são: Watznaueria barnesae, Lithastrinus floralis e Eiffellithus turriseiffeli. Estão ausentes todas as espécies características do Albiano e do Coniaciano-Santoniano.

Os sedimentos são de origem marinha. A idade Cenomaniano-Turoniano, parte mais inferior, está relacionada com a amplitude local de Classopolis major e à ocasional presença de $P$. albianus.

A distribuição geográfica da zona pode ser visualizada nă figura 4.

ZONA Marthasterites furcatus $(N-260)$ E definida pela amplitude local de Marthasterites furcatus.

As espécies mais comuns no intervalo são: Marthasterites furcatus, Lithastrinus grillii, Eiffellithus eximus, Lithastrinus floralis, Microrhabdulus stradneri, Micula staurophora, Eiffellithus turriseiffeli e Prediscosphaera cretacea.

Os sedimentos da biozona foram depositados em ambiente marinho e são de idade Coniaciano-Santoniano.

A distribuição geográfica da zona é mostrada na figura 5 .

ZONA Tetralithus ( $N$-280) A zona tem seu topo delimitado pela extinção de Tetralithus trifidus e a base pela última ocorrência de Eiffellithus eximius. As espécies mais comuns no intervalo são: Tetralithus trifidus, Broinsonia parca, Microrhabdulus stradneri, Watznaueria barnesae, Cretarhabdus? decorus, Arkhangelskiella cymbiformis, Micula staurophora, Eiffellithus turriseiffeli e Prediscosphaera cretacea. 


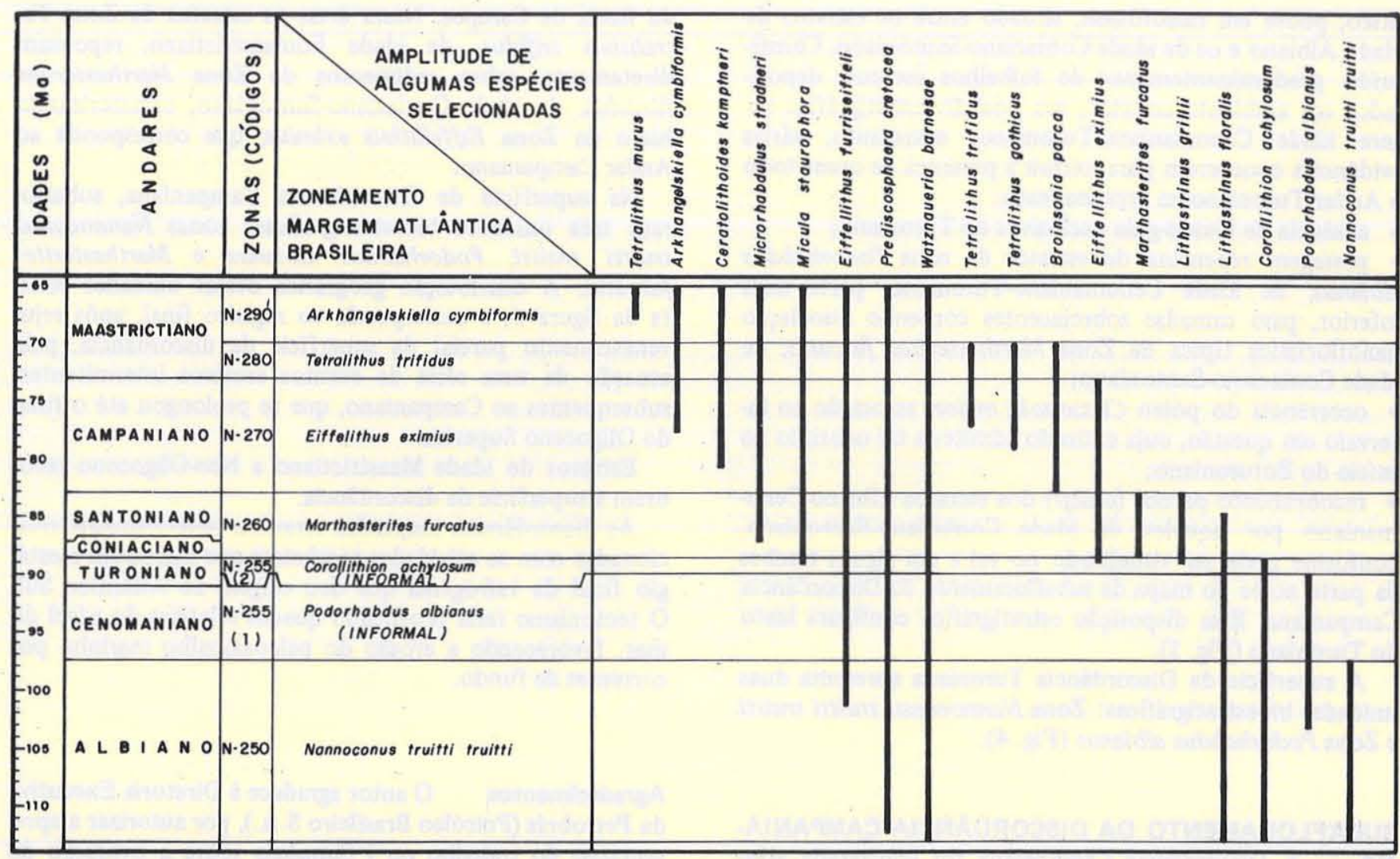

Figura 3 - Distribuição estratigráfica simplificada de nanoplâncton calcário na seqüência Albiano-Maastrictiano da margem continental brasileira. As zonas são do tipo "zona de intervalo"

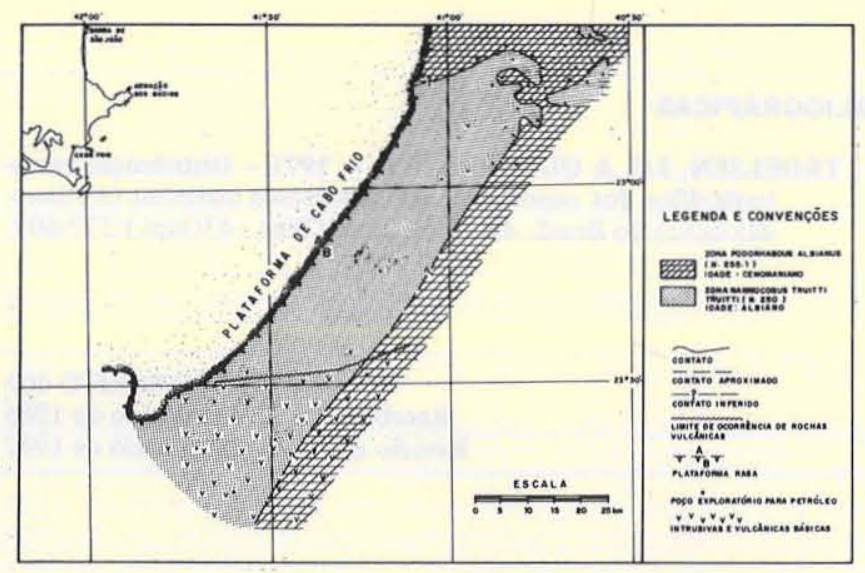

Figura 4 - Mapa de subafloramento biocronoestratigráfico da discordância turoniana no sul da Bacia de Campos

Os sedimentos da biozona foram depositados em ambiente marinho e são de idade Maastrictiano Inferior.

ZONA Arkhangelskiella cymbiformis ( $N$-290) É definida em seu topo pela extinção generalizada das espécies cretáceas. Sua base é marcada pela extinção de Tetralithus trifidus.

As espécies associadas são as mesmas relacionadas para a zona N-280, excetuando-se Tetralithus trifidus e acrescentando-se Tetralithus murus e Ceratolithoides kamptneri.

Os sedimentos da biozona são de idade Mastrictiano Superior, depositados em ambiente marinho.

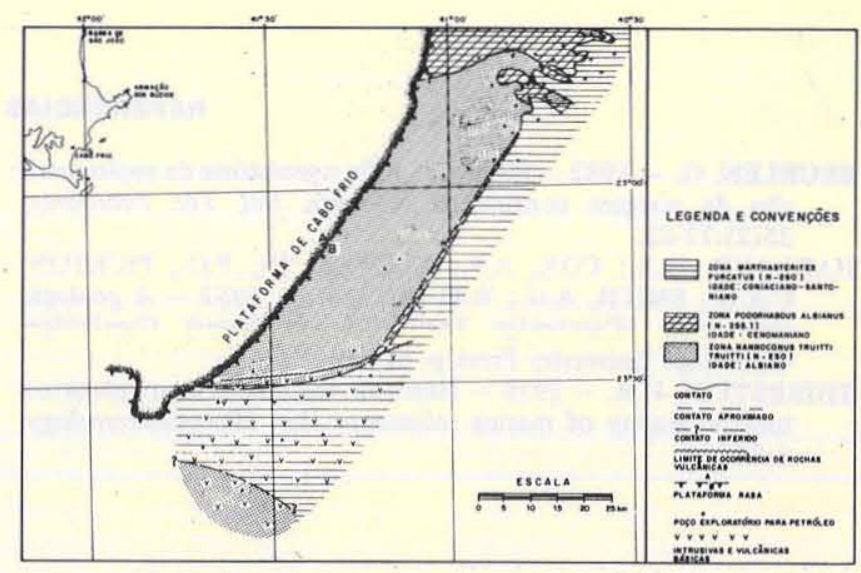

Figura 5 - Mapa de subafloramento biocronoestratigráfico da discordância campaniana no sul da Bacia de Campos

As biozonas $\mathrm{N}-280$ e $\mathrm{N}-290$ não estão representadas nos mapas das figuras 4 e 5 , pelo fato de estarem estratigraficamente acima das duas discordâncias mencionadas.

\section{SUBAFLORAMENTO DA DISCORDÂNCIA TURONIA-}

NA A Discordância Turoniana é postulada com base nos resultados das análises bioestratigráficas efetuadas em amostras de calha e testemunhos de poços exploratórios perfurados no sul da Bacia de Campos.

$\mathrm{Na}$ seqüência estratigráfica estudada, as investigações micropaleontológicas revelaram a existência de um intervalo 
lítico, pobre em nanofósseis, situado entre os estratos de idade Albiano e os de idade Coniaciano-Santoniano. Constituído predominantemente de folhelhos escuros, depositados em ambiente anóxico, sua posição estratigráfica sugere idade Cenomaniano-Turoniano; entretanto, várias evidências concorrem para excluir a presença de quase todo - Andar Turoniano na área mapeada:

- ausência de fósseis-guia exclusivos do Turoniano;

- passagem repentina de estratos da zona Podorhabdus albianus, de idade Cenomaniano-Turoniano, parte mais inferior, para camadas sobrejacentes contendo associação nanoflorística típica da Zona Marthasterites furcatus, de idade Coniaciano-Santoniano;

- ocorrência do pólen Classopolis major, associado ao intervalo em questão, cuja extinção admite-se ter ocorrido no início do Eoturoniano;

- recobrimento parcial (onlap) dos estratos Albiano-Cenomaniano por aqueles de idade Coniaciano-Santoniano, conforme pode ser visualizado no sul e em alguns trechos da parte norte do mapa de subafloramento da Discordância Campaniana. Essa disposição estratigráfica configura hiato do Turoniano (Fig. 5).

A superfície da Discordância Turoniana apresenta duas unidades bioestratigráficas: Zona Nannoconus truitti truitti e Zona Podorhabdus albianus (Fig. 4).

SUBAFLORAMENTO DA DISCORDÂNCIA CAMPANIANA A Discordância Campaniana foi constatada através de investigações bioestratigráficas, realizadas em amostras de calha de poços exploratórios perfurados no sul da Bacia de Campos. Nesta área, os estratos da Zona Tetralithus trifidus, de idade Eomaastrictiano, repousam diretamente sobre sedimentos da Zona Marthasterites furcatus, de idade Coniaciano-Santoniano, caracterizando hiato da Zona Eiffellithus eximius, que corresponde ao Andar Campaniano.

Na superfície de Discordância Campaniana, subafloram três unidades bioestratigráficas: zonas Nannoconus truitti truitti, Podorhabdus albianus e Marthasterites furcatus. A distribuição geográfica destas unidades consta da figura 5, e corresponde ao registro final, após rejuvenescimento parcial da superfície de discordância, pela atuação de uma série de eventos erosivos intermitentes, subseqüentes ao Campaniano, que se prolongou até o final do Oligoceno Superior.

Estratos de idade Maastrictiano a Neo-Oligoceno recobrem a superfície da discordância.

As discordâncias mapeadas estariam geneticamente relacionadas com as atividades tectônicas que marcaram o estágio final da tafrogenia que deu origem ao Atlântico Sul. O tectonismo teria provocado quedas relativas do nível do mar, favorecendo a erosão do paleoassoalho marinho por correntes de fundo.

Agradecimentos $\mathrm{O}$ autor agradece à Diretoria Executiva da Petrobrás (Petróleo Brasileiro S.A.), por autorizar à apresentação do trabalho no I Simpósio sobre a Evolução do Atlântico Sul, e sua publicação; ao colega Dimas Dias-Brito, pela leitura do manuscrito e valiosas sugestões.

\section{REFERÊNCIAS BIBLIOGRÁFICAS}

BEURLEN, G. - 1982 - Bioestratigrafia e geoistória da seção marinha da margem continental brasileira. Bol. Téc. Pebrobrás, 25(2):77-83.

HARLAND, W.B.; COX, A.V.; LLEWELLYN, P.G.; PICKTON, C.A.G.; SMITH, A.G.; WALTERS, R. - $1982-$ A geologic time scale (Cambridge Earth Science Series). Cambridge, Cambridge University Press. p. 31-34.

THIERSTEIN, H.R. - 1976 - Mesozoic calcareous nannoplankton biostratigraphy of marine sediments. Mar. Micropaleontology, $1: 325-62$.
TROELSEN, J.C. \& QUADROS, L.P. - 1971 - Distribuição bioestratigráfica dos nanofósseis em sedimentos marinhos (Aptiano-Mioceno) do Brasil. An. Acad. bras. Ciênc., 43(supl.):577-609.

“... Uma política mineral para a Brasil não deve ser míope com relação às transformações estruturais que estão e estarão afetando o uso de materiais, dentro do processo econômico mundial. Nisso, o desenvolvimento científico e tecnológico exercerá o papel de força motriz".

MANUSCRITO 402

Recebido em 30 de outubro de 1986 Revisão aceita em 29 de maio de 1987 